\section{Interview with James Coffman: early-life stress in adult illness}

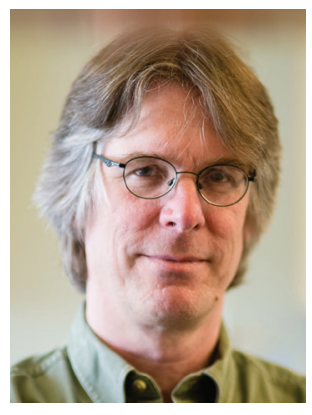

James A Coffman* speaks to Adam Price-Evans, Commissioning Editor: James A Coffman is a scientist at the MDI Biological Laboratory in Bar Harbor, ME, USA, and Director of the Maine INBRE, a NIH-sponsored program to promote biomedical research. He holds a doctorate in zoology from Duke University and a bachelor's in biology from Carleton College. He is currently studying the mechanisms by which chronic early-life stress increases adult disease risk, using zebrafish as a model organism to ask how such stress affects immune system development and regulation.

First draft submitted: 6 December 2016; Accepted for publication: 6 December 2016; Published online: 20 January 2017

\section{Q Can you give me an idea of the type of work you have been doing to date?}

I am a developmental biologist. Research in my laboratory is focused on understanding how exposure to environmental stressors during early development can affect adult anatomy and physiology. The work has two prongs. The first looks at how networks of transcriptional regulatory genes and their protein products control gene expression to direct the course of development. This is something I have been studying for over 25 years, beginning as a postdoctoral associate of the late Eric Davidson at the California Institute of Technology (CA, USA). The second prong examines how environmental variables and stressors influence the activities of transcriptional regulatory proteins that play important developmental roles, thus altering the course of development. So the work is centered on the problem of developmental plasticity.

\section{Q What first drew you toward investigating early-life stress \& its association with adult illness? \\ A few years ago I became interested in the problem of how chronic stress promotes human aging; and more specifically, the role that glucocorticoid stress signaling plays in that process. As I delved into the literature, I became intrigued by the 'fetal origins of dis- ease' hypothesis and the idea that chronic early-life exposure to elevated stress signaling can program development of phenotypes that are more prone to immune dysfunction and inflammatory disease. The mechanisms for this remain poorly understood and as a developmental biologist, I was naturally attracted to the problem.}

\section{Q What are the major issues associated with chronic exposure to psychosocial stress early in life?}

It is known that chronic psychosocial stress early in life increases disease risk later in life and possibly even in subsequent generations. A common denominator of the diseases that are predisposed to develop - ranging from asthma and rheumatoid arthritis all the way to mental health problems such as depression and anxiety - is chronic inflammation

*MDI Biological Laboratory, Kathryn W Davis Center for Regenerative Biology \& Medicine, Salisbury Cove, ME 04672, USA; jcoffman@mdibl.org

\section{KEYWORDS}

- aging • chronic stress (or 'stress')

- cortisol • developmental biology

- developmental programming

- fetal origins of disease

- glucocorticoid signaling • immune dysregulation • inflammatory disease $\bullet$ regenerative biology - zebrafish 
and immune dysregulation. So a major question is: how does chronic psychosocial stress early in life lead to chronic inflammation and immune dysregulation later in life? It is a problem of development and epigenetics.

\section{Q What is currently known about the molecular mechanisms underlying the effects of this early-life stress?}

A hypothesis that has been around for some time is that many of these effects are mediated by neuroendocrine stress signaling via the steroid hormone cortisol, which binds and thus activates the glucocorticoid receptor, a sequencespecific transcriptional regulatory protein. That is the hypothesis that we have been examining in the lab. There are many studies in the literature indicating that chronic exposure to elevated cortisol leads to glucocorticoid resistance, in at least some cases via epigenetic modification of genes such as that encoding the glucocorticoid receptor, and it is thought that this contributes to many of the health problems that ensue.

\section{Q How have you been studying the effects of this stress in your laboratory?}

Our approach has been to use zebrafish as an experimental model. The neuroendocrine stress signaling pathway in zebrafish is the same as in humans, with cortisol being the primary glucocorticoid stress hormone. Our approach has been to simply add cortisol to the culture medium (water) of developing embryos. The embryos are exposed continuously in this way to relatively low levels of cortisol $(1 \mu \mathrm{M})$ for the first 5 days of development, and then allowed to grow into adulthood normally. We assess the effects of the treatment on the embryos compared with untreated controls during and at the end of the treatment period, and later in adulthood. Our major readout thus far has been gene expression (using RNA-Seq and quantitative PCR), but we have also assessed regenerative capacity using a tail fin regeneration assay, and response to immunological challenge (LPS injection). Most recently (in [Hartig EI, Zhu S, King BL, Coffman JA, Unpublished Data]), we have used ATAC-Seq to identify genes that are epigenetically affected by the treatment.

Q What have your results revealed \& how do you think they might advance the field? Our most interesting result, recently published in Biology Open [1], is that the treated embryos develop into adults that display aberrant immune gene expression in response to injury (tail fin removal) or immunological challenge (LPS injection). This tells us two things: one, that the zebrafish, with the many advantages it provides as an experimental model for developmental biology and genetics, will provide a good model for elucidating the mechanisms through which chronically elevated glucocorticoid signaling early in life affects immunoregulation later in life; and two, our results strongly suggest that these mechanisms are epigenetic in nature, since the effects we are seeing are manifesting many months after the exposure occurred.

\section{Q How do these findings in zebrafish models relate to humans?}

We know from epidemiology that in humans chronic early life stress increases risk of developing disease later in life, and that chronic inflammation and immune dysregulation is a common feature of the diseases that develop. However, the mechanisms remain poorly understood. The fact that we see immune dysregulation in zebrafish that are chronically exposed to elevated cortisol during early development tells us that the zebrafish can be used as an experimental model to get at those mechanisms.

\section{Q How do you think this early preclinical work might be translated to benefit humans in the future $\&$ what needs to be done to achieve this?}

Ultimately, if we can elucidate the epigenetic mechanisms that mediate the effects we have observed, then we will be able to design therapies that target those mechanisms or their effects, for example, small molecules designed to reverse the adverse effects caused by specific epigenetic modifications. Beyond that, our work adds weight to the growing consensus that chronic early life stress, or even just prenatal exposure to such stress, is a major public health issue, which should motivate public policy toward mitigating those factors that contribute psychosocial adversity - for example, economic inequality, social injustice and loss of social safety nets.

\section{Q In your opinion, what are the current biggest challenges to address in this field \& what is being done to overcome these challenges?}


I think the biggest challenges are going to be disentangling the causal web through which the early exposures lead to development of disease. That web has many nodes, and there are many links in the causal chain leading from an initial exposure to a disease end point. In addition, there is a huge amount of interindividual variability, some of it genetic, some not. It is going to be difficult, if not impossible, to make sweeping generalizations, as everything is contextdependent. The field is really just scratching the surface. But I think mechanistic studies in model organisms, for example, the approach we are taking in zebrafish, will eventually lead to a deeper understanding of the epigenetic basis for developmental programming of disease risk.

\section{References}

1 Hartig EI, Zhu S, King BL, Coffman JA. Cortisoltreated zebrafish embryos develop into proinflammatory adults with aberrant immune gene regulation. Biol. Open 5, 1134-1141 (2016).

\section{Disclaimer}

The opinions expressed in this interview are those of the interviewees and do not necessarily reflect the views of Future Medicine Ltd.

\section{Financial \& competing interests disclosure}

The author's work described in the manuscript is supported by an Institutional Development Award from the National Institute of General Medical Sciences of the NIH (grant number P20-GM104318). The author have no other relevant affiliations or financial involvement with any organization or entity with a financial interest in or financial conflict with the subject matter or materials discussed in the manuscript apart from those disclosed.

No writing assistance was utilized in the production of this manuscript. 